\title{
Quench Antenna Studies of Mechanical and Quench Performance in Fermilab Interaction Region Quadrupoles for LHC
}

\author{
M. A. Tartaglia, S. Feher, A. Hocker, M. Lamm, P. Schlabach, C. Sylvester, J. C. Tompkins
}

\begin{abstract}
As part of the US-LHC collaboration, Fermilab has built and tested seventeen high gradient quadrupole magnets, assembled into nine cryostats, for installation at the Large Hadron Collider Interaction Regions. Most of these magnets have only quarter coil voltage taps for quench characterization, but the magnetic measurement warm bore is instrumented with a quench antenna for localization and characterization. We report on studies using the quench antenna for pre-production prototype (with extensive voltage taps) and 17 production magnets. These include a summary of quench localization and development characteristics, as well as general features of flux changes observed during training ramps.
\end{abstract}

Index Terms - Superconducting accelerator magnets, high gradient quadrupole, magnetic field measurement

\section{INTRODUCTION}

$\mathrm{T}$ HE production and testing of high gradient quadrupole (HGQ) magnets for LHC Interaction Regions has been in progress at Fermilab for several years as part of the US-LHC collaboration, and is nearing completion. Following the HGQ model magnet program, a full length prototype magnet MQXP01 was built and tested in 2001 [1]. Since that time, seventeen production cold masses, MQXB01-17, have been built and assembled into 9 cryostats, and tested in the Fermilab Magnet Test Facility (MTF). Quench performance results have been previously reported [2] for a subset of these magnets, known as Q2A-Q2B assemblies (with two Q2 cold masses per cryostat); MQXB03 was tested in two separate assemblies. In this paper we focus on our experience using a quench antenna for quench localization and characterization of the training quenches at $1.9 \mathrm{~K}$ for the nearly complete production series.

During the model magnet $\mathrm{R} \& \mathrm{D}$ program and prototype development, numerous voltage taps provided the primary source of quench characterization data. However, to avoid electrical problems, voltage taps are kept to a minimum in building the production magnets: all production cold masses were instrumented with quarter coil taps to identify quench locations by quadrant; MQXB01 and MQXB02, also had $8^{\text {th }}$ coil taps to distinguish inner versus outer coil quench origins.

Manuscript received September 19, 2005. This work was supported in part by the U.S. Department of Energy. M. A. Tartaglia and all co-authors are with the Fermi National Accelerator Laboratory, Batavia, IL 60510 USA (630-8403890; fax: 630-840-2383; e-mail: tartaglia@fnal.gov).
Throughout the HGQ R\&D and MQXB production magnet test programs, MTF has utilized quench antennae to provide complimentary information about the locations and development of quenches. The HGQ program utilized a 3section stationary harmonic coil probe that could be installed in the "warm bore" anti-cryostat normally used for magnetic measurements. In the production test stand, quench antenna panels were installed onto the inner surface of the insulating vacuum space of the magnetic measurement warm bore. This warm bore was installed inside the magnet beam tube, with the feed box end position and orientation fixed and a return end gasket seal that allowed for differential contraction during cool down. The entire warm bore was typically evacuated to reduce the bore temperature and system heat load, except during magnetic measurements.

\section{ANTENNA LAYOUT AND READOUT}

The antenna panels have precision traces etched on a Kapton substrate that when wrapped on the inner tube of the warm finger form a multipole winding sensitive to particular harmonics of the magnetic field. Windings have an active length of $150 \mathrm{~mm}$ and are distributed in 8 sets along the length of the magnet to detect longitudinal quench development: the lead end (LE) and body (BO) are covered by three panels and two cover the return ends (RE) of each cold mass. Sets are labeled by cold mass $(\mathrm{M} 1=\mathrm{Q} 2 \mathrm{~B}, \mathrm{M} 2=\mathrm{Q} 2 \mathrm{~A})$, and numbered by location, increasing from LE to RE. BO panels are separated by $1200 \mathrm{~mm}$, and RE panels by $20 \mathrm{~mm}$. The space between LE panels is $75 \mathrm{~mm}$, with LE1 positioned at the coil outer edge within a few millimeters; this places the LE2 panel just inside the (collared coil) body at the body-end transition.

Each antenna set has two panels with a winding on each surface: Normal (No) and skew (Sk) sextupole (Se) are on one panel with normal and skew octupole (Oc) on the other. These allow determination of the quench location and direction in the azimuthal plane as described in [3]-[4] and references therein.

Voltage signals from the 64 panel windings are conditioned by amplifiers of gain 1000 and are digitized by two 16 bit 32channel Pentek ADCs with high sample rate (ranging from 7 to $50 \mathrm{kHz}$ ). Signals are captured in a window roughly (depending upon sample rate) $270 \mathrm{~ms}$ before, and $70 \mathrm{~ms}$ after, a "quench" trigger event. A TTL-level signal into the first Pentek channel defined the trigger, created by the analog sum of the delayed 
quench protection trigger with the first quench antenna panel signal (Q2A LE1 NoSe). In this configuration it was possible, and common, for a large amplitude antenna signal to trigger the data loggers.

\section{FluX ChANGE EVENTS}

\section{A. Large Amplitude Oscillation Events}

During the first production magnet tests, we became aware of the self-triggering issue when the quench antenna logger captured data at high current during a ramp, but the magnet had not yet quenched and other loggers had not been triggered. Often several such events were captured during the first ramp to quench, but never on subsequent ramps. In a few cases, this prevented capture of antenna data during an actual quench. It became clear that these triggers resulted from large oscillating signals in all panels that spontaneously appeared in the Q2A magnets. Fig. 1 shows an example of NoOc signals from one such event - signal amplitudes in LE are greatest, but decline and are delayed with distance from the LE. Study of data

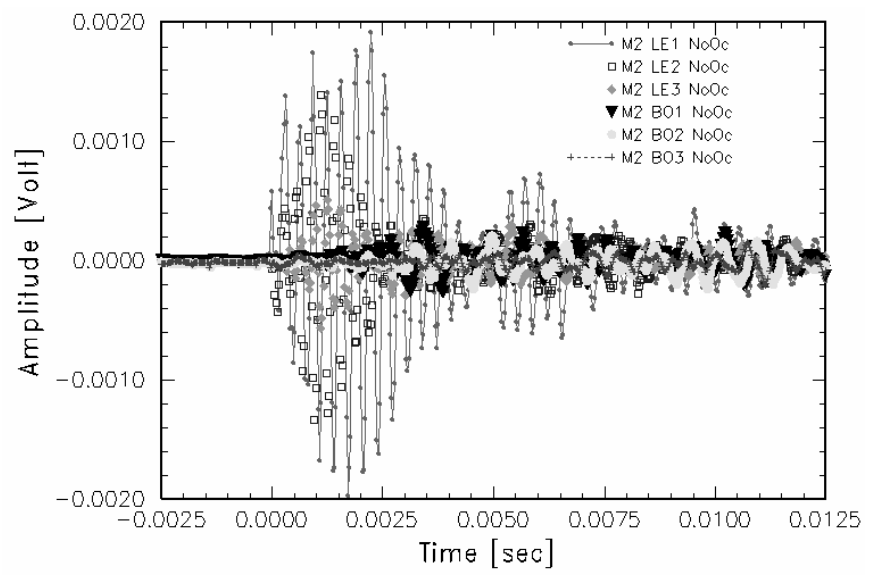

Fig. 1. Self-triggered quench antenna event captured at $9750 \mathrm{~A}$ on first ramp far below quench current, NoOc signals for magnet MQXB14 (Q2A).

captured during quenches shows that similar events occur at random in all regions of the $\mathrm{Q} 2 \mathrm{~A}$ and $\mathrm{Q} 2 \mathrm{~B}$ magnets. Since they appear not to have caused any quenches, we believe these are the result of some motion of the warm bore during the first ramp (e.g. stick/slip along the beam tube after thermal cycle), which causes the warm bore and antenna panels to vibrate in the magnetic field. Antenna data was captured by randomly triggering with the warm bore open and magnet powered, and oscillations with a similar spectrum are seen. Also, identical self-triggered events can be induced by lightly tapping the end of the warm bore with the magnet powered.

\section{B. Small Amplitude Transient Events}

In the data captured for all production magnet training quenches, there is a great deal of transient flux change activity visible across the magnet. In contrast to the large amplitude signals discussed earlier, these are much smaller and very brief events: typically 0.1 to $1 \mathrm{mV}$ amplitude, and $0.5 \mathrm{~ms}$ duration. Fig. 2 shows the NoSe signals for all panels in the window captured just before a typical training quench. In magnets that have experienced spurious quench detection system triggers at high current after having been trained to a higher current, the quench antenna shows a dramatically lower level of activity. It seems reasonable to conclude that these transients are each caused by mechanical "training" motions across the magnet,

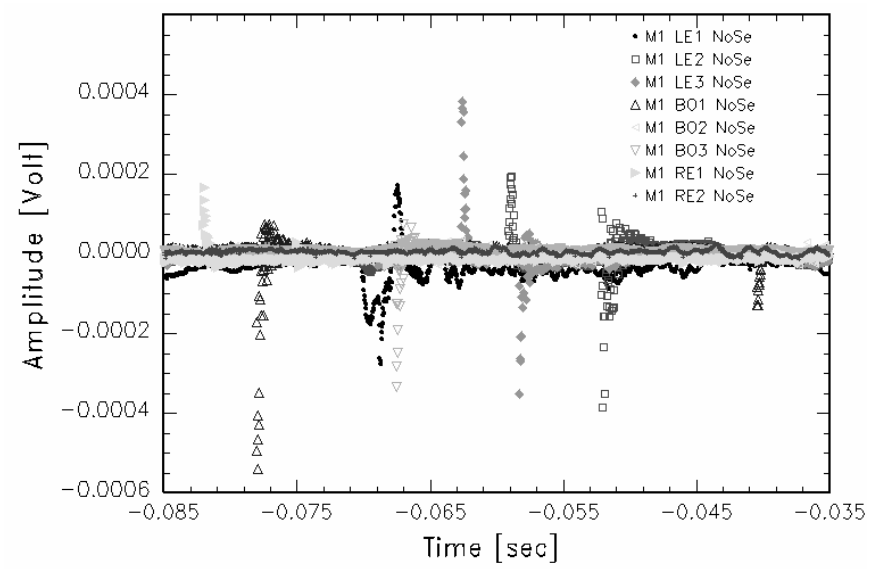

Fig. 2. Typical Example of Transient Flux-change events (NoSe signals shown) captured in time window leading up to the second training quench in MQXB05.

which are not sufficiently energetic or suitably located to cause a quench.

\section{QUENCH LOCALIZATION}

\section{A. Antenna Calibration}

Two of the production cold masses, MQXB04 and MQXB14, did not train and did not reach the target operating current of 12208 A. Great focus was therefore placed upon systematic study of the available quench antenna data in trying to assess the underlying reason for the limitations. To localize a propagating quench front, the antenna analysis makes an underlying assumption [4] about a moving line current redistribution that results in flux changes in the antenna. The azimuthal angle $\alpha$ and radius $\mathrm{R}$ at which this redistribution occurs in our detector, in which all panels have the same length and number of windings, are defined by (1) and (2); Vns and Rns represent the NoSe voltage and panel radius, etc.

$$
\begin{gathered}
\alpha=\tan ^{-1}\left[\left(\frac{V s s}{V n s}\right)\left(\frac{R n s}{R s s}\right)^{3}\right]-\tan ^{-1}\left[\left(\frac{V s o}{V n o}\right)\left(\frac{R n o}{R s o}\right)^{4}\right] \\
R=\sqrt{\frac{\left(V n s /\left[3 R n s^{3}\right]\right)^{2}+\left(V s s /\left[3 R s s^{3}\right]\right)^{2}}{\left(V n o /\left[4 R n o^{4}\right]\right)^{2}+\left(V s o /\left[4 R s o^{4}\right]\right)^{2}}}
\end{gathered}
$$

The results are quite sensitive to ratios of voltages that change quickly with angle, and our initial results were somewhat confusing. Therefore we performed a series of calibrations of the antenna response to flux changes induced by external currents. This was done using a mechanical fixture to precisely locate a wire with respect to individual antenna panels, parallel to the antenna axis at known radius and azimuth, the wire excited with a current source, and antenna data captured. The fixture was clamped to the outer warm bore 
tube, positioned over a wide range of angles each precise to about 1 degree relative to the warm bore alignment mark. A wire in the fixture was positioned at $34 \mathrm{~mm}$, and another at 48 $\mathrm{mm}$ radius, to map the response as a function of radius at each angle. Data were captured while the wires were individually excited by a square wave-driven current source, thereby inducing flux changes to step-function line current changes. Each of the Q2A and Q2B LE antenna sets were calibrated in this manner, and the resulting voltage signals were plotted and fitted to obtain offset angles and amplitude coefficients according to the expected sinusoidal response of each harmonic coil to a line current source [5]. The known wire radius allows us to constrain the relative $\mathrm{Oc}$ to $\mathrm{Se}$ amplitude for each set of panels. BO and RE panel sets have not yet been fully calibrated, but are not as compelling as the LE regions.

We discovered by this effort several important things: first, the M1 LE1 panel NoOc and SkOc panel had a large angle offset of about 22 degrees, while all others had a small offset of 2 or 3 degrees; in hindsight, such an assembly error is possible with the panel alignment marks. Second, we found unexpectedly large gain variations $(\sim 20 \%)$ between panels. Although "direct" amplifier calibrations showed variations of only a percent, the system response to flux changes appears sensitive to the wiring arrangement, probably from some impedance mismatch. Calibrations were repeated and results were reproducible. However, re-analysis of data from the prototype and first production magnet have not been made because cabling arrangements were different in those tests.

\section{B. Azimuthal Resolution}

The application of calibration data made tremendous improvement in the consistency of calculated quench positions in the azimuthal plane. It allowed verification of the Q2A,Q2B coordinate systems in space, and of voltage tap quadrant indications. The high sample rate data permit averaging of points in small time segments (to reduce effects of noise), and we observe segment to segment consistency within a couple of degrees in angle, and a couple of millimeters in radius. Similar consistency is also seen between adjacent panels (in the LE). We have not yet explored the use of data filters to reduce noise.

\section{Inner versus Outer}

Voltage taps in the HGQ models, MQX prototype and first production magnet identify which training quenches occurred in the outer coil. Only two prototype and no LQXB01 quenches originated in the outer coil. In the former case, antenna signals appear only in one quench: the LE1 antenna signals arise a few milliseconds after voltage rise begins, at the same time the quench appears in the inner coil. Reviewing HGQ09 test data, two outer coil-only quenches clearly show quench antenna signals arising simultaneously with the outer coil voltages. Therefore, the inner superconducting coil does not necessarily screen current redistribution fluxes from the antenna, although further study would be necessary to determine whether azimuthal and radial analysis are affected.

\section{QUENCH DEVELOPMENT}

\section{A. Longitudinal Origin and Development}

Table 1 summarizes the quench development of production TABLE I QUENCH LOCATIONS

\begin{tabular}{|c|c|c|c|c|c|c|c|c|c|}
\hline Assembly & Q2 & Cold mass & $\begin{array}{l}\text { Quench } \\
\text { No. }\end{array}$ & $\begin{array}{l}\text { Quench } \\
\text { Current }\end{array}$ & $\begin{array}{c}\text { Quadrant } \\
\text { No. }\end{array}$ & $\begin{array}{l}\text { Longitudinal } \\
\text { Location }\end{array}$ & $\begin{array}{l}\text { Precursor } \\
\text { to V(coil) }\end{array}$ & $\begin{array}{l}\text { Azimuthal } \\
\text { Location* }\end{array}$ & Comment $* *$ \\
\hline \multirow[t]{2}{*}{ LQXB01 } & $\mathrm{A}$ & MQXB02 & 1 & $12710 \mathrm{~A}$ & Q3Inner & $\mathrm{LE} 1,2$ & LE1 & N/A & \\
\hline & & & 2 & $12955 \mathrm{~A}$ & Q1Inner & LE1 & -- & N/A & \\
\hline LQXB02 & $\mathrm{B}$ & MQXB04 & $\begin{array}{c}1 \\
2-18\end{array}$ & $\begin{array}{c}11152 \mathrm{~A} \\
--\end{array}$ & $\begin{array}{l}\mathrm{Q} 2 \\
\mathrm{Q} 2\end{array}$ & LE1, 2 & -- & $\mathbf{N R}(\mathrm{LE} 1,2,3)$ & $\begin{array}{l}\text { LE1 moves } \mathbf{R} \\
\text { all } \sim \text { Identical }\end{array}$ \\
\hline \multirow[t]{2}{*}{ LQXB03 } & B & MQXB05 & 1 & $12495 \mathrm{~A}$ & $\mathrm{Q} 2$ & LE2 & LE2 & $\mathbf{N R}(\mathrm{LE} 1,2,3)$ & \\
\hline & & & 2 & $12905 \mathrm{~A}$ & Q2 & LE2 & LE2 & $\mathbf{N R}(\mathrm{LE} 1,2,3)$ & Identical to $1 \mathrm{st}$ \\
\hline \multirow[t]{4}{*}{ LQXB04 } & B & MQXB12 & 1 & $12097 \mathrm{~A}$ & Q2 & LE1 & -- & $\mathbf{R}(\mathrm{LE} 1), \mathbf{N R}(\mathrm{LE} 2,3)$ & LE1 moves NR \\
\hline & & & 2 & $12445 \mathrm{~A}$ & Q1 & $\mathrm{LE} 1,2,3$ & LE2 & $\mathbf{R}(\mathrm{LE} 1,2,3)$ & EO \\
\hline & & & 3 & $12749 \mathrm{~A}$ & Q3 & $\mathrm{LE} 1,2$ & LE2 & $\mathbf{R}(\mathrm{LE} 1,2,3)$ & Similar to 2 nd \\
\hline & & & 4 & $12850 \mathrm{~A}$ & Q2 & $\mathrm{BO} 2$ & -- & N/A & \\
\hline \multirow[t]{3}{*}{ LQXB05 } & A & MQXB11 & 1 & $12236 \mathrm{~A}$ & Q4 & (BO?) & -- & & $\mathrm{NF}$ \\
\hline & & & 2 & $12974 \mathrm{~A}$ & Q3 & (BO?) & -- & & $\mathrm{NF}$ \\
\hline & B & MQXB08 & 1 & $12922 \mathrm{~A}$ & Q3 & LE1,2,3 & LE2 & $\mathbf{R}(\mathrm{LE} 1,2,3)$ & EO, LE moves $\mathbf{N R}$ \\
\hline \multirow[t]{4}{*}{ LQXB06 } & A & MQXB07 & 1 & $11827 \mathrm{~A}$ & Q4 & no antenna data & -- & & \\
\hline & & & 2 & $12538 \mathrm{~A}$ & Q4 & LE1, 2 & LE1, 2 & $\mathbf{R}(\mathrm{LE} 1,2,3)$ & $\mathrm{EO}$ ? \\
\hline & & & 3 & $12812 \mathrm{~A}$ & Q4 & LE1, 2 & LE1, 2 & $\mathbf{R}(\mathrm{LE} 1,2,3)$ & $\sim$ Identical to $2 \mathrm{nd}$ \\
\hline & B & MQXB09 & 1 & $12864 \mathrm{~A}$ & Q1 & $\mathrm{BO} 3$ & -- & N/A & \\
\hline \multirow[t]{5}{*}{ LQXB07 } & A & MQXB14 & 1 & $10864 \mathrm{~A}$ & Q2 & LE1,2 & -- & $\mathbf{N R}(\mathrm{LE} 1,2,3)$ & LE1 moves $\mathbf{R}$ \\
\hline & & & $2-5$ & -- & Q2 & & & & all Identical \\
\hline & B & MQXB15 & 1 & $12634 \mathrm{~A}$ & Q2 & (BO?) & -- & & $\mathrm{NF}$ \\
\hline & & & 2 & $12831 \mathrm{~A}$ & Q2 & (BO?) & -- & & $\mathrm{NF}$ \\
\hline & & & 3 & $13063 \mathrm{~A}$ & Q4 & $\mathrm{BO} 1,2,3$ & -- & N/A & Warm Bore Open \\
\hline LQXB08 & $\mathrm{B}$ & MQXB13 & 1 & $12817 \mathrm{~A}$ & Q1 & LE1 & LE2 & $\mathbf{P O}(\mathrm{LE} 1,2), \mathbf{U}(\mathrm{LE} 3)$ & Ringing Signals \\
\hline \multirow[t]{3}{*}{ LQXB09 } & $\mathrm{B}$ & MQXB16 & 1 & $12427 \mathrm{~A}$ & Q2 & LE1, 2 & LE1,2 & $\mathbf{U}(\mathrm{LE} 1,2)$ & Ringing LE3 \\
\hline & & & 2 & $12702 \mathrm{~A}$ & Q3 & LE2, 3 & LE2 & $\mathbf{P O}(\mathrm{LE} 1,2,3)$ & \\
\hline & & & 3 & $12790 \mathrm{~A}$ & Q1 & LE3 & LE3 & $\mathbf{R}(\mathrm{LE} 2,3)$ & No LE1 quench \\
\hline
\end{tabular}

* N/A=Not Analyzed, U=Unlocalized, R=Ramp side, NR=NonRamp side, $\mathrm{PO}=$ Pole center ** EO=Extended Origin, NF=Not Found 
magnet training quenches, ramping at $20 \mathrm{~A} / \mathrm{s}$ in $1.9 \mathrm{~K}$ superfluid helium. Five of the seventeen magnets did not quench below their target current. No training quenches occurred in the RE of any magnet. In two magnets (four quenches), quench antenna signals did not arise in any set, and these are presumed to have been $\mathrm{BO}$ quenches. Three BO quenches were detected in three magnets; the last of these (MQXB15) followed magnetic measurements with the warm bore open, adding additional heat to the system, and the quench occurred almost simultaneously in all three BO sets.

Most of the quenches, then, originated in the LE region. Excluding the two limited magnets, there are 10 quenches in 5 magnets. In three magnets, signal development in successive training events are strikingly similar in all details, at very different currents. Also, in eight quenches, for five magnets, one or more of the LE quench antenna sets show large flux change signals at least one millisecond before resistive voltage growth begins in the coils; typically the quench development begins in more than one antenna simultaneously, in some cases accompanied by a kind of ringing. Most often the first signals are seen in LE1 and/or LE2, at the Body/End transition. These features suggest that mechanical events in the end may have initiated these quenches.

In the two limited magnets, many quenches of each magnet were recorded: the currents and development features exhibit

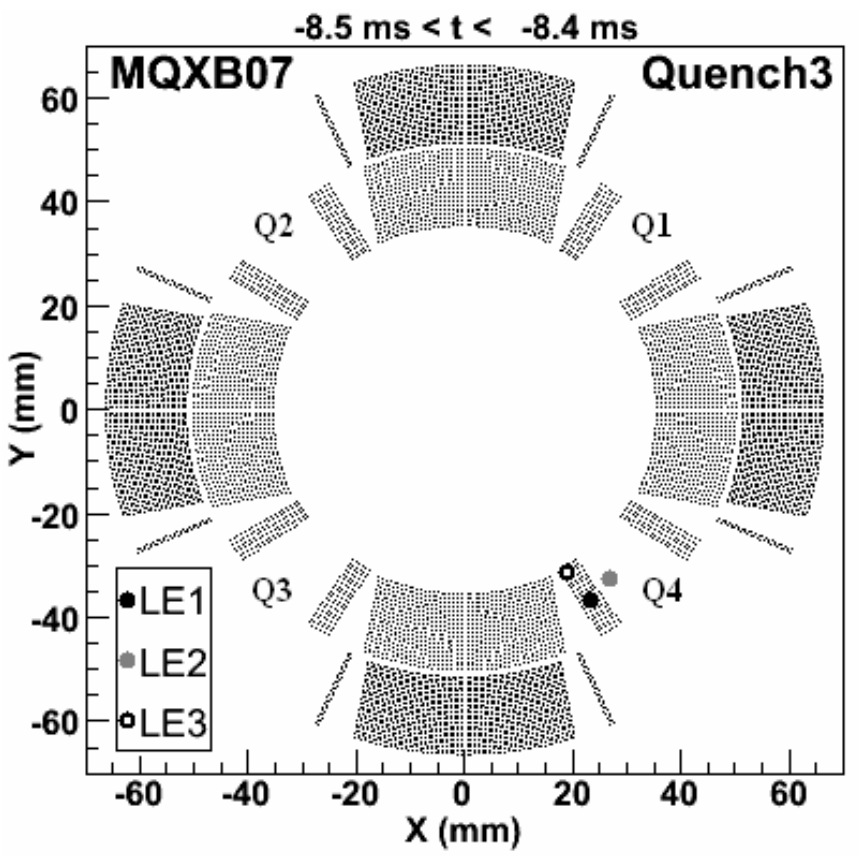

Fig. 3. Coil cross section viewed from Lead End with calculated quench locations, averaged over $0.1 \mathrm{~ms}$ time slice. In this example all three LE antennae point to quadrant Q4 Ramp Side Inner Coil pole turns 12-14.

remarkable reproducibility. In both cases, the origin is near the LE1-LE2 boundary, and the antenna signals arise simultaneously with coil resistive voltage. The quenches then propagate through LE2 and appear in LE3 soon afterward.

Quench velocity is difficult to determine with the available segmentation - "time of flight" between panels is a possibility, but the precise quench origin is usually not known. When upper or lower limits can be estimated they are consistent with calculations based upon rate of resistive voltage rise.

\section{B. Radial and Azimuthal Development}

We have not yet studied BO quench azimuthal characteristics. For quenches that developed in the LE, we find the quench always develops through at least two of the antennae. For most of those the quench starting locations appear to be consistent and stable, and they point to the inner coil turns 12-14, which are located between the wedge and the pole and experience the highest magnetic fields. Fig. 3 shows the coil cross section of as viewed from the magnet Lead End, the inner/outer ramp turn is just to the clockwise side of the pole; turns just counter-clockwise are on the "non-ramp" side.

Particularly in the LE1, quench locations often start on one side of the pole, but then move to the middle of the pole or jump to the other side; this may be because the antenna covers the turn around the coil ends, or because there is a quench developing on both sides of the pole.

Not all quenches are amenable to analysis under the assumption of a single redistribution current. In a number of cases, the analysis indicates quench locations centered on the pole or inside the inner coil radius, rather than pointing towards a conductor. Often, these have very "disorganized" rather than "nicely developing" signals, as if there is a superposition of multiple redistributing currents inducing flux changes. Many antenna signals have features - particularly polarity transitions and "waves" in amplitude - that suggest multiple quench fronts may be developing.

\section{CONCLUSION}

The quench antenna provides a unique window into the workings of superconducting magnets. Further study of strength and direction of redistributing currents is planned. Analysis of quench behavior versus temperature and ramp-rate are possible, and two new cold masses will be tested soon.

\section{ACKNOWLEDGMENT}

The authors gratefully acknowledge helpful discussions with T. Ogitsu (KEK) and A. Devred (CEA/CERN), and thank the Fermilab Magnet Test Facility staff for their committed efforts.

\section{REFERENCES}

[1] N.Andreev, et al., "Quench performance and mechanical behavior of the first fermilab-built prototype high gradient quadrupole for the LHC interaction regions," IEEE Trans. Appl. Supercond. vol. 12, pp. 59-62, March 2002.

[2] R.Bossert, et al., "Test results of LHC interaction region quadrupoles produced by Fermilab," IEEE Trans. Appl. Supercond. vol. 15, pp. 10901093, June 2005.

[3] T.Ogitsu, et al., "Quench antenna for superconducting particle accelerator magnets," IEEE Trans. Magnetics vol. 30, pp. 2273-2276, 1994.

[4] T.Ogitsu, et al., "Quench observation using quench antennas on RHIC IR quadrupole magnets," IEEE Trans. Magnetics vol. 32, pp. 3098-3101, 1996.

[5] A.K.Jain, Basic Theory of Magnets, CERN Accelerator School (CAS 97): "Measurement and alignment of accelerator and detector magnets," Anacapri, Italy, Apr 1997. 